\title{
Análise clínica e biomecânica do efeito do diclofenaco sódico na consolidação da fratura da tíbia no rato*.
}

\author{
Clinical and biomechanical analysis of the effect of diclofenac sodium in tibial fracture \\ healing in rats*
}

Sérgio Swain Müller ${ }^{1}$, Emillo Carlos Curcelli ${ }^{2}$, Trajano Sardenberg ${ }^{2}$

Alexandre Zuccon ${ }^{3}$, José Luiz De Crudis Júnior ${ }^{3}$, Carlos Roberto Padovani ${ }^{4}$

\section{RESUMO}

Os AINH (Antiinflamatórios não hormonais) são agentes utilizados na prática clínica que interferem no processo inflamatório pela inibição da síntese de prostaglandinas e tromboxanos. Alguns trabalhos experimentais investigaram sua ação no processo de consolidação de fraturas, por meio de estudos clínicos e histológicos, sendo escassas as análises biomecânicas. Nesse estudo foram utilizados 20 ratos da linhagem Wistar, divididos aleatoriamente em dois grupos iguais: grupo A (controle) e grupo B (tratado com diclofenaco sódico). Em ambos os grupos foram realizadas fraturas abertas, após perfuração, na tíbia direita. A administração da droga foi via intramuscular, dose única diária, por 28 dias. Os animais foram pesados semanalmente. Após o sacrifício as tíbias foram dissecadas, pesadas e submetidas a ensaio biomecânico de flexão analisando-se carga máxima, deformação e coeficiente de rigidez. Observou-se que no grupo tratado com AINH não houve aumento do peso corpóreo a partir da segunda semana e as tíbias fraturadas foram mais pesadas. Neste grupo o calo ósseo suportou menor carga máxima, apresentando maior deformação e menor coeficiente de rigidez. Nos animais tratados, o osso não fraturado também se mostrou menos rígido. Concluiu-se, nas condições estudadas, que o DS alterou o processo de consolidação e o metabolismo ósseo, levando a retardo na maturação do calo e menor rigidez do osso intacto, respectivamente.

Descritores: Antiinflamatório; Consolidação da fratura; Ratos.

\section{SUMMARY}

The antinflammatories are agents utilized on clinical practice that interfere on inflammatory process by synthesis inhibition of prostaglandin and tromboxanes. Some experimental studies investigated their action on the fractures consolidation process, through clinical and histological studies, as the biomechanical analyses are scarce. In this study, 20 (twenty) Wistar pedigree rats were used, aleatory divided into two groups: A group (control) and B group (treated with diclofenac). In both groups open fractures were made through perforation on right tibia. The drug administration was done intramuscularly in a single daily dose, during 28 (twenty-eight) days. The animals were weekly weighed. After the sacrifice the tibias were dissected, weighed and submitted to a biomechanical analysis of flexion and the maximum load, deformation and rigidity coefficient were measured. It was remarkable that the treated group with antinflammatory did not present body weight gain after the second week and the broken tibias were heavier. In this group the osseous callous supported less maximum load, presenting larger deformation and smaller rigidity coefficient. In the treated group, the non-fractured bone was also less rigid. Concluding, at this studied conditions, the diclofenac can interfere on the consolidation process and on the osseous metabolism, promoting a delay on the callous maturation and a smaller rigidity of the intact bone, respectively.

Keywords: Antiinflammatory; Fracture healing; Rats
Trabalho realizado no Departamento de Cirurgia e Ortopedia (DCO) da Faculdade de Medicina de Botucatu (UNESP), Universidade Estadual Paulista "Júlio de Mesquita Filho".

1 - Professor Assistente Doutor do DCO, FMB - UNESP

2 - Professor Assistente do DCO, FMB - UNESP

2 - Professor Assistente do DCO, FMB - UNESP

4 - Professor Titular do Depto. de Bioestatística, IB - UNESP

Endereço para correspondência: Departamento de Cirurgia e Ortopedia Faculdade de Medicina de Botucatu - UNESP Botucatu - São Paulo - Cep: 18.618.970 e-mail:ssmuller@uol.com.br

Trabalho recebido em04/02/2003. Aprovado em 25/07/2004
Work performed at the Department of Surgery and Orthopedics of the Medicine School of (UNESP), Paulista State University "Júlio de Mesquita Filho".

1 - Assistant Professor PHD at the Department of Surgery and Orthopedics, FMB UNESP

2 - Assistant Professor at the Department of Surgery and Orthopedics, FMB -UNESP 3 - Ex-Resident at the Service of Orthopedics and Traumatology, FMB-UNESP 4 - Chairman of the Biostatistical Department - UNESP

Address: Department of Surgery and Orthopedics Medicine School of Botucatu - UNESP Botucatu - São Paulo - e-mail:ssmuller@uol.com.br 


\section{INTRODUÇÃO}

O potencial de reparação de um osso fraturado é dependente de variáveis relacionadas ao paciente, localização e também ao tratamento empregado. O tratamento deve criar condições biológicas e mecânicas que favorecem a reparação. É evidente que as características do paciente e da fratura impõem limites à cicatrização, mas dentro desses limites, o tratamento pode influenciar a cura ${ }^{(3)}$.

O tecido ósseo é único no organismo dos vertebrados na capacidade de reparação sendo possível a restauração biológica e mecânica, diferentemente de outros órgãos e tecidos ${ }^{(7)}$.

Diversos fatores podem influenciar a organização e resultado final do processo de consolidação de uma fratura, positivamente ou negativamente. Alguns desses fatores exercem influência que só pode ser investigada em estudos experimentais ${ }^{(3)}$.

A cicatrização dos tecidos músculo-esqueléticos ocorre por meio de resposta tecidual composta de 3 fases, que não acontecem separadamente. Essas fases podem ser descritas como inflamação, reparação e remodelação.

A resposta inflamatória é considerada indispensável ao processo de reparação. Sem a inflamação, os tecidos jamais cicatrizariam $^{(5,28)}$.

Os antiinflamatórios não hormonais (AINH) são freqüentemente usados na prática clínica em pacientes com traumas, fraturas, pós-operatório, etc, pelo efeito analgésico.

As drogas AINH têm atividade antiinflamatória, analgésica e antipirética, sendo que a maioria pode inibir a função plaquetária. É aceito que essas drogas exerçam efeitos farmacológicos pela inibição da ciclooxigenase, que modula a síntese de alguns mediadores da inflamação, como as prostaglandinas ${ }^{(2,9)}$.

Entre os diversos AINH utilizados atualmente, destaca-se o diclofenaco sódico (DS) que é antiinflamatório não hormonal derivado do ácido fenilacético, indicado para uso em condições dolorosas crônicas e agudas, inflamação pós-operatória e póstraumática, aliviando a dor e diminuindo a reação inflamatória e o edema ${ }^{(16,27)}$.

A maioria dos efeitos antiinflamatórios do DS são devidos à inibição da síntese de prostaglandinas, sendo potente inibidor da ciclooxigenase in vivo e in vitro, além de diminuir a síntese de prostaglandinas, também diminui a síntese de tromboxano e prostaciclina $^{(18,26,27)}$

Vários autores descreveram retardo de consolidação em tíbias de ratos tratados com AINH, por meio de estudo histológi$\mathrm{CO}^{(1,12)}$

A análise biomecânica do calo ósseo tem sido referida como um dos melhores métodos de avaliação da consolidação de fraturas por diversos autores ${ }^{(6,8)}$.

Poucos trabalhos experimentais relacionaram a consolidação de fraturas e o uso de AINH analisando propriedades biomecânicas. A prescrição rotineira dessas drogas em pacientes com traumas agudos motivou o presente estudo experimental com um dos antiinflamatórios mais usados na prática clínica atual, o diclofenaco sódico.

\section{INTRODUCTION}

Fracture repair potential depends upon several factors, including those related to the patient, fracture site, and treatment.

Treatment aims to enhance fracture repair by improving biological and mechanical conditions.

Patient and fracture characteristics restrict healing. However, treatment may have an effect upon cure ${ }^{[3]}$.

In contrast with other tissues and organs, bone tissue is characterized by a singular repair potential, biological and mechanical repair being possible ${ }^{[7]}$.

Different factors can positively or negatively affect the organization and outcome of fracture repair. Growth factors, demineralized matrix, bone marrow cells ${ }^{[3]}$.

Healing of musculoskeletal tissues is a tissular response consisting of three phases that do not occur separately, namely, inflammation, repair, and remodeling.

Inflammatory response, therefore, is essencial for repair. Tissues would not heal in the absence of inflammation ${ }^{[5,28]}$.

Nonsteroidal antiinflammatory drugs (NSAIDs) are often used in clinical practice in patients with trauma, fractures, following surgery, etc because of their analgesic effect.

NSAIDs have antiinflammatory, analgesic, and antipyretic effects. Most of these agentes can inhibit platelet function.

Their pharmacological effects are due to cyclooxygenase inhibition. This enzyme modulates the synthesis of some inflammation mediators, such as prostaglandins ${ }^{[2,9]}$.

Several NSAIDs are used today. Diclofenac sodium (DS), a commonly used NSAID, is derived from phenylacetic acid and indicated in the management of acute and chronic painful conditions, postoperative and posttraumatic inflammation, because it relieves pain and decreases inflammatory reaction and edema ${ }^{[16,27] .}$

The majority of antiinflammatory effects of DS are due to inhibition of prostaglandin synthesis. DS is a potent inhibitor of cyclooxygenase in vivo and in vitro. It decreases the synthesis of prostaglandins and also of thromboxane and prostacyclin [18, 26, 27].

Several authors have described delayed fracture repair in tibias in histological studies in NSAIDs-treated rats ${ }^{[1,12]}$.

Biomechanical analysis of bone callus has been considered one of the best methods to evaluate fracture repair ${ }^{[6,8]}$.

Biomechanical properties have been studied in few experimental studies evaluating fracture repair and NSAIDs use.

The routine use of NSAIDs in patients with acute trauma has led us to carry out the present experimental study using one of the most widely used antiinflamatory agents in the current clinical practice, diclofenac sodium. 


\section{MATERIAL E MÉTODO}

Foram utilizados 20 ratos da linhagem Wistar (Rattus norvegicus albinus), com 60 dias de idade, peso médio de 253g, clinicamente sadios. Por sorteio, foram formados dois grupos de 10 animais: grupo A (controle) e grupo B (tratado com diclofenaco sódico).

O experimento foi desenvolvido em seis fases seqüenciais: adaptação; fratura; constituição dos grupos; manutenção e tratamento; sacrifício e ensaio mecânico.

Os animais foram submetidos inicialmente a exame clínico, identificação, pesagem, e em seguida, alojados em gaiolas, em local seco e arejado, limpo diariamente e com temperatura controlada. Receberam água e alimento ad libitum.

Após sete dias de adaptação, os animais foram examinados, pesados e anestesiados com pentobarbital sódico, na dose $30 \mathrm{mg} / \mathrm{kg}$, via intraperitoneal ${ }^{(25)}$. Utilizando-se técnicas assépticas, foi produzida perfuração no ponto médio da tíbia direita, utilizando-se furadeira elétrica, com broca de $1 \mathrm{~mm}$ e rotação controlada por pedal. Concluída a perfuração, a broca foi retirada e a fratura completada manualmente ${ }^{(14)}$.

Após a fratura, por sorteio, foram constituídos os grupos.

Os animais foram então recolocados nos alojamentos, examinados diariamente e pesados em intervalos de sete dias e no dia do sacrifício. Não foi utilizado qualquer tipo de imobilização, permitindo-se livre movimentação do membro fraturado. A administração da droga foi iniciada 12 horas após a fratura e repetida a cada 24 horas, na dose de $10 \mathrm{mg} / \mathrm{kg} / \mathrm{dia}$, via intramuscular.

Após 28 dias da produção da fratura todos os animais foram submetidos ao exame físico geral, exame do membro fraturado (quanto à mobilidade do foco) e pesagem; foram então sacrificados pela técnica de decapitação e ambas as tíbias foram removidas.

A análise efetuada foi clínica e biomecânica, compreendendo a tíbia fraturada e a não-fraturada em ambos os grupos. Para a análise clínica foram considerados: peso semanal, comportamento dos animais e eventual presença de complicações como infecção, formação de hematomas ou alterações gastrintestinais.

Para a retirada das tíbias, foi realizada incisão ampla, longitudinal, na região anterior do membro do animal. A seguir, a tíbia foi desarticulada proximal e distalmente. A fíbula foi removida e foram retiradas as estruturas musculares, capsulares e tendíneas em toda a extensão restando, portanto, apenas o tecido ósseo (Figura 1). Em seguida, a tíbia foi pesada em balança analítica de precisão de quatro casas após a vírgula e capacidade para

\section{MATERIAL AND METHODS}

Twenty clinically healthy Wistar rats (Rattus norvegicus albinus) aged 60 days were used. Mean weight was $253 \mathrm{~g}$. Two groups of 10 animals each were defined by draw. Group A was used as control and Group B included diclofenac-treated rats.

The experimental study had six sequential phases: adaptation; fracture; group formation; maintenance and treatment; sacrifice; and mechanical assay.

Animals were submitted to clinical examination, identification, weighing and confined in cages in a dry and ventilated area. Cages were cleaned daily. Temperature was controlled. Animals were given water and food ad libitum.

Following 7-day adaptation animals were examined, weighed, and anesthetized with sodium pentobarbital at the dose of 30 $m g / k g$ given intraperitoneally ${ }^{[25]}$.

Under aseptical conditions a perforation was drilled at the mean point of the right tibia using an electric dril with a $1 \mathrm{~mm}$-drill and pedal-controlled rotation.

After perforation drill was removed and fracture was manually completed ${ }^{[14]}$. Groups were defined by draw following fracture. Animals were then confined in a cage.

They were examined daily and weighed at 7-day intervals and on the sacrifice day. Free motion of the fractured limb was allowed, immobilization being not used. Intramuscular diclofenac sodium (10 mg/kg/day) was given $12 \mathrm{~h}$ following fracture and every $24 \mathrm{~h}$ thereafter.

All animals were submitted to general physical examination, examination of the fractured limb (as for fracture focus mobility) and weighing 28 days after fracture. They were sacrificed by decapitation and both tibias were removed.

Clinical and biomechanical analyses of fractured and nonfractured tibias were carried out. Clinical analysis included weekly weight, behavior, eventual complications, such as infection, hematoma, or gastrointestinal disorders.

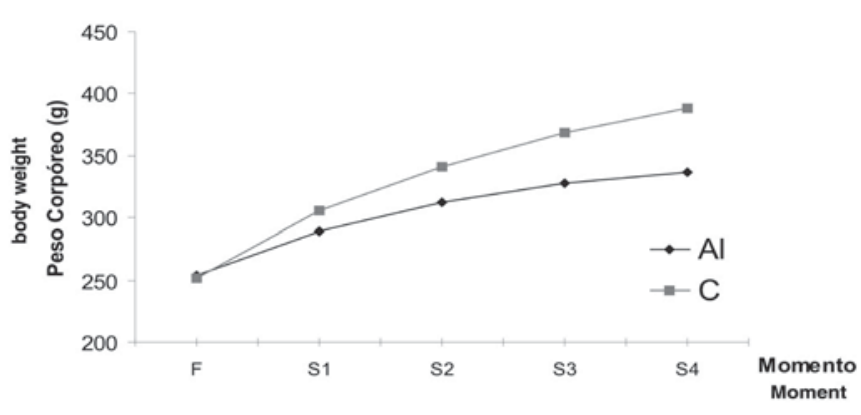

Figura 1 - Média do peso corpóreo (g) segundo grupo e momento de avaliação

Figure 1 - Body weight mean (g) accordng to study group and timepoint
A longitudinal incision was made on the anterior face of the limb for tibial removal by proximal and distal desarticulation.

Fibula and muscular, capsular, tendinous structures were completely removed. (Figure 1).

Only bone tissue remained in the specimen. Tibias were then weighed with high-precision analytical scales (4 decimals) of 110 -gm capacity. Specimens were stored in plastic bags at -20 ${ }^{\circ} \mathrm{C}$ (a domestic refrigerator was used). 
110 gramas. As peças foram acondicionadas em sacos plásticos e conservadas em congelador doméstico $\left(-20^{\circ} \mathrm{C}\right)$

No dia da realização dos ensaios, as peças foram descongeladas em temperatura ambiente, envolvidas em compressas umedecidas.

Foi então realizado ensaio de flexão de três pontos com a máquina universal de ensaios tipo eletromecânica (EMIC, DL 10000), em ambiente com temperatura controlada e estável (20 C). A precisão do sistema é de $\pm(0,018+$ F 3700) KN, conforme especificações da norma NBR 6674 (ABNT, 1981).

O corpo de prova foi colocado sem fixação, com a face convexa voltada para cima, sobre dois suportes eqüidistantes dos pólos proximal e distal da tíbia, mantendo distância, equivalente a 2/3 do comprimento do osso. Um cutelo de ponta romba ( $2 \mathrm{~mm}$ de largura), fixo ao cabeçote proximal da máquina de ensaios, transmitiu a carga que foi aplicada no ponto eqüidistantes aos dois apoios. A carga foi aplicada até ruptura do corpo de prova $^{(8,24)}$, com velocidade de $30 \mathrm{~mm} / \mathrm{min}$ (figura 2).

O relatório final do ensaio forneceu o valor da carga máxima e deformação na ruptura, além do diagrama carga-deformação. Calculou-se em cada diagrama o valor da constante de proporcionalidade (K) ou coeficiente de rigidez.

A análise comparativa do peso corpóreo, peso do osso, carga máxima, deformação e coeficiente de rigidez, nos dois grupos experimentais analisados, foi realizada pela técnica da análise multivariada dos perfis médios e pela técnica ANOVA não-paramétrica para o modelo de medidas repetidas com dois grupos independentes ${ }^{(15,20)}$.

Todas as conclusões estatísticas foram discutidas no nível de $5 \%$ de significância.

\section{RESULTADOS}

\subsection{Avaliação Clínica}

Os animais tratados com DS apresentaram distúrbio gastrintestinal (diarréia) no início do experimento (5 primeiros dias) e obtiveram menor ganho de peso a partir da segunda semana.

Todos os ratos realizaram apoio do membro progressivamente desde a realização da fratura.

A avaliação clínica das tíbias demonstrou mobilidade na região do calo no grupo de ratos tratados com o diclofenaco de sódio.

As Tabelas 1, 2, 3 e a Figura 3 apresentam os dados relacionados ao peso dos animais e ao peso das tíbias.
On the day when assays were carried out specimens were thawed at room temperature and wrapped in moist drapes.

A three-point flexion assay was carried out with an electromechanical-type universal assay machine (EMIC, DL 10000) under controlled temperature $\left(20^{\circ} \mathrm{C}\right)$. System precision is $\pm(0,018+$ F 3700) KN, according to NBR 6674 specifications (ABNT, 1981).

The specimen with the convex side facing upward was placed on two supports equally distant from the proximal and distal tibial ends. No fixation was used and distance corresponded to $2 / 3$ of bone length. A blunt knife $(2 \mathrm{~mm})$ fixed to the assay machine applied the load on the equidistant point until the specimen was broken [8, 24] (speed: $30 \mathrm{~mm} /$ min)(Figure 2).

Values of maximum load and deformation upon breakage as well as the load-deformation graph are included in the final assay report. The proportionality coefficient $(K)$ or rigidity coefficient was calculated for each diagram.

Body weight, bone weight, maximum load, deformation, and rigidity coefficient were compared by multivariate analysis of mean profiles and non-parametric ANOVA for repeated measurements in two independent groups [15, 20].

Statistical analysis adopted a significance level of 5\%.

\section{RESULTS}

\subsection{Clinical Evaluation}

Diclofenac-treated animals developed gastrointestinal disorders (diarrhea) during the first five days. Their body weight gain was smaller from the second week on, as compared to controls.

All rats progressively supported the limb following tibial fracture.

Clinical evaluation of tibias has shown mobility of bone callus in diclofenac-treated rats.

Animal and tibia weight data are shown in Tables 1, 2, 3, and Figure 3.

\subsection{Mechanical Properties}

Mechanical property data are shown in Tables 4, 5, and 6 
3.2 Propriedades Mecânicas

As tabelas 4,5 e 6 apresentam os valores das propriedades mecânicas avaliadas.

As figuras 4 e 5 representam os diagramas carga-deformação nos ossos com e sem fratura.

\section{DISCUSSÃO}

No presente estudo experimental, optou-se por utilizar ratos como animais de experimentação, principalmente, por serem de pequeno porte, baixo custo ${ }^{(21)}$ e de consolidação óssea rápida com ou sem tratamento ${ }^{(19)}$ (em torno de 28 dias para as fraturas da tíbia), além de serem freqüentemente utilizados como modelos em estudos de consolidação de fraturas, entre outras vantagens.

Os antiinflamatórios não hormonais (AINH) podem provocar alterações no metabolismo ósseo e na consolidação de fraturas, provavelmente por alterarem a fase inflamatória do processo de consolidação(17). Ao inibirem as ciclooxigenases, diminuem a síntese de mediadores inflamatórios, entre estes as prostaglandinas, que têm como principal função, na fase inicial do processo de consolidação, exercer ação quimiotática e dessa forma diminuir a quantidade de células no foco de fratura, diminuindo também a reabsorção do tecido necrótico e possivelmente alterando a quantidade de células de reparação, cruciais para a formação do calo ósseo ${ }^{(4)}$.

Optou-se pelo diclofenaco sódico (DS) por ser um dos AINH mais utilizados na prática clínica e também estudado em animais de experimentação. A dose terapêutica preconizada para ratos é de 2 - $10 \mathrm{mg} / \mathrm{kg} / \mathrm{dia}$, podendo ser administrada via intramuscular, intraperitoneal ou subcutânea(11).

Utilizou-se, portanto, a dose máxima terapêutica do DS ${ }^{(4)}$ (10 $\mathrm{mg} / \mathrm{kg} / \mathrm{dia}$ ) durante 28 dias (tempo estimado para consolidação da fratura) procurando estudar o eventual máximo efeito durante todo o processo de consolidação. and load-deformation diagrams for fractured and non-fractured bones are shown in Figures 4 and 5 .

\section{DISCUSSION}

In the present experimental study rats were used as experimental animals, mainly because they are small and cheap [21] and develop fast bone repair with or with treatment [19] (approximately 28 days for tibial fracture). In addition, they are often used in models of fracture repair, among other advantages.

Nonsteroidal antiinflammatory drugs (NSAIDs) can lead to changes in bone metabolism and fracture repair, probably because they affect the inflammatory phase of healing [17].

By inhibiting cyclooxygenases they decrease the synthesis of inflammatory mediators, including prostaglandings responsible for chemotaxis durign the first phase of fracture healing, thus reducing the amount of cells in the fracture site and reabsorption of necrotic tissue and possibly modifying the amount of repair cells essential for bone callus formation ${ }^{[4]}$.

Diclofenac sodium (DS) was chosen because it is one of the most widely used NSAIDs in clinical practice and has been studied in laboratory animals.

Therapeutical dosage recommended for rats ranges from 2 to $10 \mathrm{mg} / \mathrm{kg} / \mathrm{day}$. It can be given intramuscularly, intraperitoneally, and subcutaneously ${ }^{[11]}$;

In the present study, the maximal therapeutical dose of DS ${ }^{[4]}$ (10 $\mathrm{mg} / \mathrm{kg} /$ day) was used for 28 days (estimated time required for a fracture to heal).

The expected maximal effect was studied throughout fracture repair.

We chose the experimental model of fracture following open perforation because this technique has been used in similar stu- 
Dentre os diversos modelos experimentais de fratura, escolheu-se a fratura após perfuração aberta, pois além de ser a técnica já utilizada em nosso serviço para estudos do gênero, permite controle do local da fratura e menor força aplicada(14,19).

A análise biomecânica do calo de fratura é reportada por diversos autores como uma das formas de se avaliar a consolidação óssea ${ }^{(6,8)}$.

Analisando os resultados, observou-se que o grupo de animais tratado com AINH não apresentou ganho ponderal a partir da segunda semana, fato que pode ser associado à ação destas drogas na inibição da síntese protéica ${ }^{(10)}$. Alteração gastrintestinal (diarréia), identificada nesse grupo, também pode ter contribuído com tal achado clínico. $\mathrm{Na}$ literatura esse efeito colateral é mencionado em doses acima de 10 mg/kg/dia ${ }^{(11)}$.

Com relação à média do peso das tíbias fraturadas, constatou-se maior valor no grupo tratado com DS, observação que poderia ser explicada pela maior formação tecidual por provável instabilidade do calo(22).

A avaliação biomecânica foi realizada por meio da comparação dos seguintes parâmetros: carga máxima, deformação absoluta e coeficiente de rigidez.

Observou-se que os corpos de prova dos ratos tratados com AINH suportaram menor carga de maneira significativa antes da ruptura do calo ósseo e também apresentaram maior deformação, conseqüentemente gerando menor coeficiente de rigidez. O AINH reduzindo a síntese de prostaglandinas e tromboxanos provavelmente alterou o processo de neoangiogênese, determinando menor aporte de oxigênio às células mesenquimais. Nessas condições essas células apresentariam tendência à diferenciação em condro-



Figura 3 - Diagrama carga $x$ deformação construído com os valores das medianas obtidas nos ensaios mecânicos das tíbias do lado esquerdo (sem fratura)

Figure 3 - Load versus deformation diagram based upon median values obtained upon mechanical assays of left tibias (non-fractured)

\begin{tabular}{|c|c|c|c|}
\hline \multirow{2}{*}{$\begin{array}{c}\text { Grupo } \\
\text { Group }\end{array}$} & \multicolumn{2}{|c|}{ Lado / Side } & \multirow{2}{*}{$\begin{array}{c}\text { Resultado do teste } \\
\text { de fratura } \\
\text { Result of the fracture teste }\end{array}$} \\
\cline { 2 - 3 } & $\begin{array}{c}\text { Direito (fratura) } \\
\text { Right (fracture) }\end{array}$ & $\begin{array}{c}\text { Esquerdo (controle) } \\
\text { Left (control) }\end{array}$ & $2,70(\mathbf{p}<0,01)$ \\
\hline $\begin{array}{c}\text { Antiinflamatório } \\
\text { NSAID }\end{array}$ & $18,9(9,6-58,7) a A$ & $53,9(50,8-72,1) \boldsymbol{a B}$ & $2,80(\mathbf{p}<0,01)$ \\
\hline $\begin{array}{c}\text { Controle } \\
\text { Control }\end{array}$ & $31,0(17,1-45,8) \boldsymbol{b A}$ & $65,0(56,4-95,6) \boldsymbol{b B}$ & \\
\hline $\begin{array}{c}\text { Resultado oo testo de grupo } \\
\text { Result of group test }\end{array}$ & $1,96(\mathbf{p}<0,05)$ & $2,79(\mathbf{p}<0,01)$ & \\
\hline
\end{tabular}

$a, b, A, B$ : letras diferentes indicam diferença estatística (minúsculas vertical; maiúsculas horizontal) $a, b, A, B$ different letters indicate a statistical difference (lowercase letters vertical; capital letters horizontal) Tabela 4 - Mediana e valores mínimo e máximo da carga máxima $(N)$ e respectivos resultados do teste estatístico.

Table 4 - Median and minimal and maximal values of maximum load (N) and respective statistical results.

\begin{tabular}{|c|c|c|c|}
\hline \multirow{2}{*}{$\begin{array}{l}\text { Grupo } \\
\text { Group }\end{array}$} & \multicolumn{2}{|c|}{ Lado / Side } & \multirow{2}{*}{$\begin{array}{l}\text { Resultado do teste } \\
\text { de fratura } \\
\text { Result of the fracture teste }\end{array}$} \\
\hline & $\begin{array}{l}\text { Direito (fratura) } \\
\text { Right (fracture) }\end{array}$ & $\begin{array}{l}\text { Esquerdo (controle) } \\
\text { Left (control) }\end{array}$ & \\
\hline $\begin{array}{l}\text { Antiinflamatório } \\
\text { NSAID }\end{array}$ & $2,6(1,9-4,4) b B$ & $1,9(1,5-2,1) a A$ & $2,43(\mathbf{p}<0,05)$ \\
\hline $\begin{array}{c}\text { Controle } \\
\text { Control }\end{array}$ & $1,5(0,6-2,6)$ aA & $1,7(1,4-2,4) a A$ & $1,07(\mathbf{p}>0,015$ \\
\hline 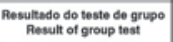 & $1,79(\mathbf{p}<0,05)$ & $0,08(\mathbf{p}>0,05)$ & \\
\hline
\end{tabular}

$a, b, A, B$ : letras diferentes indicam diferença estatística (minúsculas vertical; maiúsculas horizontal) $a, b, A, B$ : different letters indicate a statistical difference (lowercase letters vertical; capital letters horizontal) Tabela 5 - Mediana e valores mínimo e máximo de deformação na carga máxima $(\mathrm{mm})$ e respectivos resultados do teste estatístico.

Table 5 - Median and minimal and maximal values of deformation upon maximum load $(\mathrm{mm})$ and respective statistical results.

\begin{tabular}{|c|c|c|c|}
\hline \multirow{2}{*}{$\begin{array}{c}\text { Grupo } \\
\text { Group }\end{array}$} & \multicolumn{2}{|c|}{ Lado / Side } & \multirow{2}{*}{$\begin{array}{c}\text { Resultado do teste } \\
\text { de fratura } \\
\text { Result of the fracture teste }\end{array}$} \\
\cline { 2 - 3 } & $\begin{array}{c}\text { Direito (fratura) } \\
\text { Right (fracture) }\end{array}$ & $\begin{array}{c}\text { Esquerdo (controle) } \\
\text { Left (control) }\end{array}$ & $2,80(\mathbf{p}<0,01)$ \\
\hline $\begin{array}{c}\text { Antiinflamatório } \\
\text { NSAID }\end{array}$ & $11,5(5,40-28,60)$ aA & $41,9(37,6-56,9) \mathbf{a B}$ & $2,49(\mathbf{p}<0,05)$ \\
\hline $\begin{array}{c}\text { Controle } \\
\text { Control }\end{array}$ & $27,9(20,7-57,50)$ bA & $54,1(38,0-66,0) \mathbf{b B}$ & \\
\hline $\begin{array}{c}\text { Resultado do tosto do grupo } \\
\text { Alessutt of group tost }\end{array}$ & $3,36(\mathbf{p}<0,01)$ & $2,64(\mathbf{p}<0,01)$ & \\
\hline
\end{tabular}

$a, b, A, B$ : letras diferentes indicam diferença estatística (minúsculas vertical; maiúsculas horizontal) $a, b, A, B$ different letters indicate a statistical difference (lowercase letters vertical; capital letters horizontal) Tabela 6 - Mediana e valores mínimo e máximo do coeficiente de rigidez $(\mathrm{N} / \mathrm{mm})$ e respectivos resultados do teste estatístico.

Table 6 - Median and minimal and maximal values of rigidity coefficients $(\mathrm{N} / \mathrm{mm})$ and respective statistical results. dies in our institution, allows one to control the fracture site, and requires less force ${ }^{[14,19]}$

The biomechanical analysis of the fracture callus has been considered by several authors a technique of bone repair evaluation ${ }^{[6,8]}$.

In diclofenac-treated animals body weight gain was not seen from the second week on. This finding can be related to the $D S$ effect upon protein synthesis inhibition [10] as well as to diarrhea seen in DS-treated animals. This side effect has been described in literature when doses higher than $10 \mathrm{mg} / \mathrm{kg} / \mathrm{day}$ have been used ${ }^{[11]}$.

As for the mean weight of fractured tibias, greater values were found in DS-treated animals, which could be explained by greater tissue formation due to probable callus instability [22].

Biochemical analysis evaluated maximum load, absolute deformation, and rigidity coefficient.

Tibial specimens from DStreated rats supported significantly lower load before bone callus rupture and showed greater deformation and lower rigidity coefficients than control animals. NSAIDs-induced decreased prostaglandin and thromboxane synthesis probably affected neoangiogenesis, less oxygen being supplied to mesenchymal cells. Under these conditions there would be a tendency to differentiation into chondroblasts and fibroblast responsible for extraceIlular matrix synthesis with no calcium affinity. Therefore, an immature and less mineralized bone callus would develop [1].

The evaluation of mechanical properties of non-fractured tibias showed lower rigidity coefficients in DS-treated 
blastos e fibroblastos responsáveis pela síntese de matriz extracelular sem afinidade pelo cálcio e caracterizando-se, portanto, provável calo ósseo imaturo e menos mineralizado ${ }^{(1)}$.

A avaliação das propriedades mecânicas das tíbias não fraturadas também revelou menor coeficiente de rigidez no grupo de ratos tratados com DS. Essa alteração poderia ser explicada pela influência dos AINH não somente na consolidação, mas também na homeostase óssea de maneira a estimular a ação reabsortiva dos osteoclastos $^{(13)}$.

Ro et al. ${ }^{(23)}$ em estudo biomecânico de consolidação de fraturas em fêmur de ratos tratados com indometacina demonstraram menor resistência do calo ósseo.

Allen et al(1) realizaram avaliação histológica de regenerados ósseos em ratos tratados com indometacina e aspirina, observaram maior porcentagem de união cartilaginosa nesses animais, tendo sido demonstrado retardo no processo de consolidação.

Keller et al. ${ }^{(17)}$ verificaram o montante de fluxo de sangue em calos ósseos de coelhos tratados com indometacina seguido de avaliação biomecânica. Constatou-se menor aporte sangüíneo ao calo nas fases iniciais da consolidação nos animais tratados e diminuição da carga máxima em comparação ao grupo controle.

Em estudo recente, Giordano et al. ${ }^{(12)}$ confirmaram histologicamente as observações desses autores. Verificaram atraso na ossificação do calo ósseo, na fratura de rato tratado com tenoxicam.

\section{Considerações Clínicas}

A análise dos resultados da presente investigação e da literatura existente mostram evidências do possível efeito deletério dos AINH no processo de reparação óssea. Diante do exposto o senso comum parece indicar que a utilização de AINH em casos de fratura, quando e se necessário, deve ser feita de maneira criteriosa, pelo menor tempo possível, e apenas nos

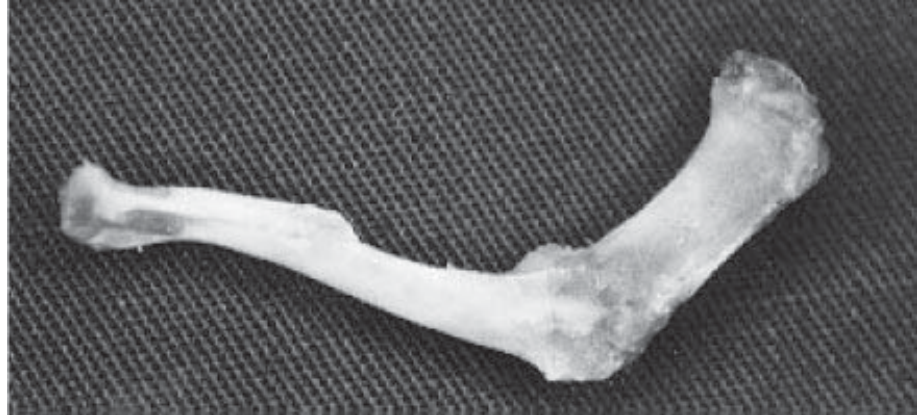

Figura 4 - Aspecto da tíbia após dissecção mostrando o calo de fratura. Figure 4 - View of tibia after dissection showing the callus fracture.
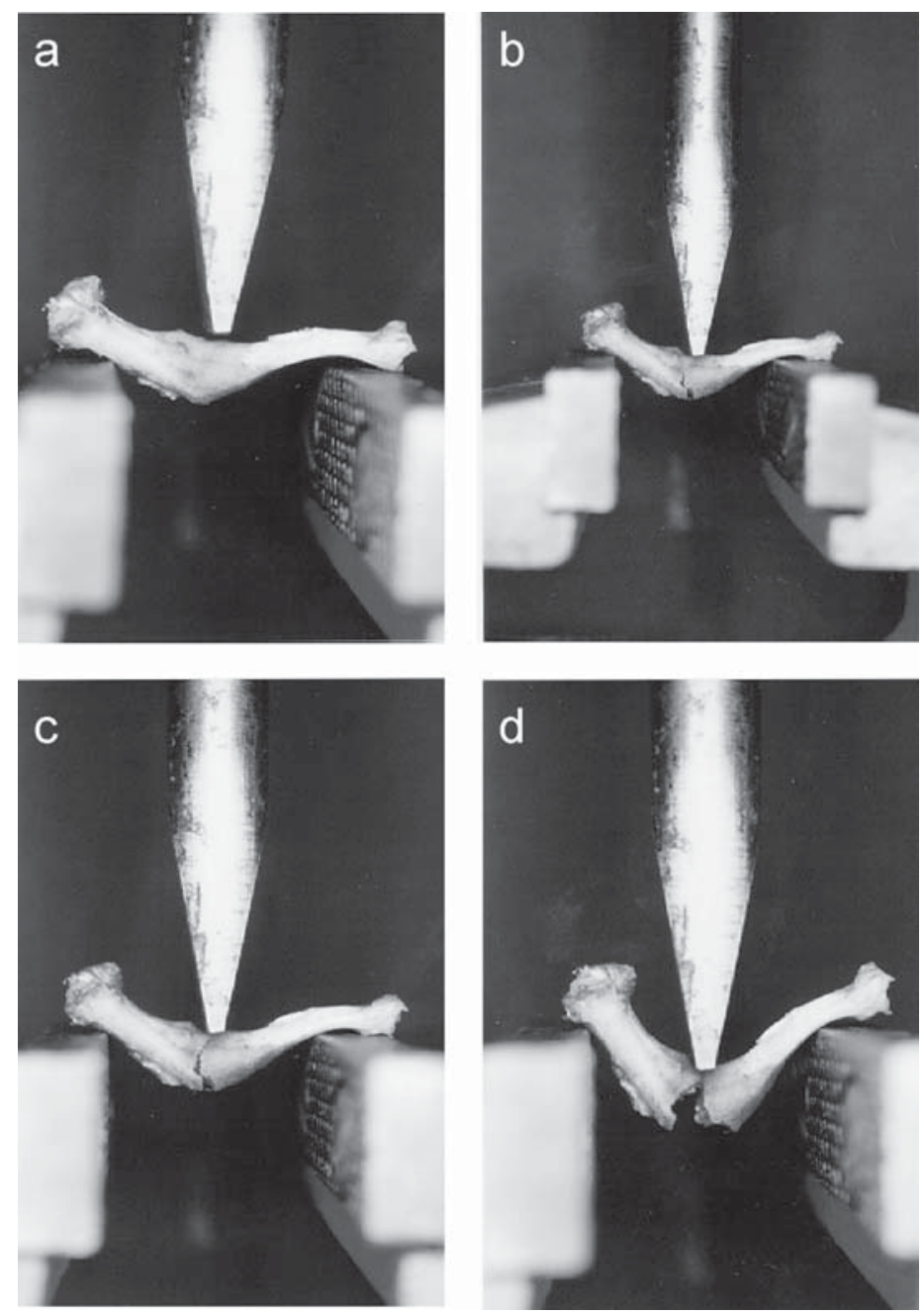

Figura 5 (a-d) - Tíbia, com fratura, montada na máquina universal de ensaio. Seqüencia do ensaio de flexão até a ruptura do corpo de prova.

Figure 5 (a-d)- Tibia, with fracture, arranged in the universal teste machine. Sequence of bending test until the rupture of the bone. rats, a finding that could be explained by the NSAID effect upon not only fracture repair, but also upon bone homeostasis so as to stimulate reabsorptive action of osteoclasts ${ }^{[13]}$.

In a biomechanical study of femoral fracture repair in indomethacin-treated rats Ro et al. ${ }^{[23]}$ showed less resistant bone callus.

Allen et al. ${ }^{[1]}$ histologically studied regenerated bone in indomethacin- and aspirin-treated rats and observed a higher percentage of cartilaginous union in these animals, thus confirming delayed fracture healing.

Keller et al. [17] observed blood flow in bone callus in indomethacin-treated rabbits and carried out a biomechanical evaluation.

Less blood was supplied to bone callus during the first phases of bone repair in treated animals, as compared to animals. Another finding was decreased maximum load

In a recent study Giordano et al. ${ }^{[12]}$ histologically confirmed Keller's findigns. They also confirmed delayed ossification of bone callus in tenoxicam-treated rats.

\section{Clinical Considerations}

Literature reports and the present study show evidence of a possible deleterious effect of NSAIDs upon bone healing.

Therefore, NSAIDs should be carefully indicated in patients with fracture only for short-term therapy and only if trauma-related symptoms require their use. Patients should be instructed to avoid self-medication. 
casos em que a prescrição seja indispensável para o controle de eventuais sintomas relacionados ao traumatismo. Os pacientes devem também ser orientados no sentido de evitar a auto medicação.

\section{CONCLUSÕES}

No rato e nas condições observadas:

- O DS inibiu o ganho de peso corpóreo;

- O peso do osso fraturado foi maior nos animais tratados;

- O DS reduziu o coeficiente de rigidez do osso não fraturado;

- O DS alterou as propriedades mecânicas do calo ósseo diminuindo a Carga Máxima e Rigidez e aumentando a deformação.

Esses achados permitiram concluir que houve retardo de consolidação óssea no grupo de animais tratados com diclofenaco sódico.

\section{CONCLUSIONS}

Under study conditions the following has been observed in rats:

- DS inhibited body weight gain;

- The weight of fractured bone was greater in treated ani mals;

- DS reduced the rigidity coefficient in non-fractured bones;

- DS affected mechanical properties of bone callus, decreasing maximum load and rigidity and increasing deformation.

These findings confirm delayed bone repair in diclofenac-treated animals.

\section{REFERÊNCIAS BIBLIOGRÁFICAS}

1. Allen HL, Wase A, Bear WT. Indomethacin and aspirin: effect of non-steroidal anti-inflammatory agents on the rate of fracture repair in the rat. Acta Orthop Scand 51: 595-600, 1980

2. Brooks PM, Day RO. Non steroidal antinfalmmatory drugs: differences and similarities. N Engl J Med 324: 1716-1725, 1991.

3. Buckwalter JA, Cruess RL. A cura dos tecidos musculoesqueléticos. In: Rockwood Jr CA, Green DP, Bucholz RW. eds. Fraturas em adultos. 3a ed. São Paulo: Manole, 1993. p.179-219.

4. Cerqueira NF. Efeito do diclofenaco de sódio sobre a cicatrização de anastomoses término-terminais em artérias carótidas em coelhos: avaliação anatomo-patológica e da força de ruptura. [Tese]. Botucatu-SP, Brasil, Faculdade de Medicina Veterinária e Zootecnia, 2000. 160p

5. Cotran RS, Kumar U, Robbins SL. Inflamação e reparação. In: Robbins SL, Patologia estrutural e funcional. 5a ed. Rio de Janeiro: Guanabara Koogan 1996. p. 45-83.

6. Delgado-Martinez AD, Martinez ME, Carrascal MT et al. Effect of 25-OH-vitamin D on fracture healing in elderly rats. J Orthop Res 16: 650-653, 1998

7. Einhorn TA. The cell and molecular biology of fracture healing. Clin Orthop 355: 7-21, 1998.

8. Ekeland A, Engesaeter LB, Langeland N. Mechanical properties of fractures and intact rat femura evaluated by bending, torsional and tensile tests. Acta Orthop Scand 52: 605-613, 1981.

9. Faraj M, Rabelo HC, Gontijo MB. Tópicos atuais sobre o uso de antinflamatórios não esteróides na clínica. J Bras Med 73: 57-69, 1997.

10. Fatureto MC, Gomes RAS, Hial V, Teixeira VPA, Goldenberg S. Curva ponderal pós-operatória em ratos tratados com diclofenaco sódico. Acta Cir Bras 6 : 41-43, 1991.

11. Fatureto MC. Poder de sustentação de feridas fechadas e parâmetros da cicatrização em ratos tratados com diclofenaco sódico. [Tese]. São Paulo, Brasil, Escola Paulista de Medicina, 1990. 186p.

12. Giordano V, Giordano M, Knackfuss I.G, et al. Influência do tenoxicam no processo de consolidação de fratura de tíbia. Estudo experimental em ratos. Rev Bras Ortop 34: 395-400, 1999.

13. Head JE, Bryant BJ, Grills BL, Ebeling PR. Effect of short-term use of ibuprofen or acetoaminophen on bone resorption in healthy men: a double-blind, placebo-controlled pilot study. Bone 29: 437-441, 2001.
14. Jackson RW, Reed CA, Israel JA, Abou-Keer FK, Garside H. Production of a standard experimental fracture. Can J Surg 13: 415-420, 1970.

15. Johnson RA, Wichern DW. Applied multivariate statistical analysis. $3^{\mathrm{a}}$ ed. New Jersey: Preutice Hall, 1992. 642p.

16. Kantor TG. Use of diclofenac in analgesia. Am J Med 80: 64-69, 1986.

17. Keller J, Bünger C, Andreassen TT, Bak B, Lucht U. Bone repair inhibited by indomethacin effects on bone metabolism and strength of rabbit osteotomies. Acta Orthop Scand 58: 379-383, 1987

18. Ku EC, Wasvary JM, Cash WD. Diclofenac sodium (GP 45840, Voltaren), a potent inhibitor of prostaglandin synthetase. Biochem Pharmacol 24: 641-643, 1975.

19. Müller SS. Efeito da subnutrição na consolidação da fratura da tíbia no rato: estudo radiológico, anátomo-patológico e bioquímico. [Tese]. Botucatu-SP, Brasil, Faculdade de Medicina de Botucatu (UNESP), 1991. 175p.

20. Norman GR, Streiner DL. Biostatistics - The bare essentials. 1a ed. Saint Louis: Mosby Book, 1994. 260p.

21. Nunamaker DM. Experimental models of fracture repair. Clin Orthop 355: 5665, 1998.

22. Ro J, Langeland N, Sander J. Effect of indomethacin on collagen metabolism of rat fracture callus in vitro. Acta Orthop Scand 49: 323-328, 1978.

23. Ro J, Sudmann E, Marton P F. Effect of indomethacin on fracture healing in rats. Acta Orthop Scand 47: 588-599, 1976.

24. Sammarco GJ, Burstein A, Davies W, Frankel V. The biomechanics of torsional fractures: the effect of loadind on ultimate properties. J Biomech 4: 113-117, 1971.

25. Sardenberg T. Estudo histológico da artroplastia de ressecção com e sem interposição de novelo tendinoso. Modelo experimental em cães. [Tese]. Botucatu-SP, Brasil: Faculdade de Medicina de Botucatu (UNESP), 1997. 115p

26. Takayama F, Egashira T, Kudo Y, Yamanaka Y. Effects of anti-free radical interventions on phosphatidylcholine hydroperoxide in plasma after ischemia-reperfusion in the liver of rats. Biochem Pharmacol 46: 1749-1757, 1993.

27. Todd PA, Sorkin EM. Diclofenac sodium: a reppraisal of its pharmacodynamic and pharmacokinetic properties and therapeutic efficacy. Drugs 35: 244-285, 1988.

28. Wahl SM. Fibrosis: bacterial-cell-wall-induced hepatic granulomas. In: Gallin F.I., Goldstein I.M., Snyderman R. eds. Inflamation: basic principles and clinical correlates. 1a ed. New York: Raven Press, 1988. p.841-860. 